\title{
Non-inferiority of retrospective data collection for assessing perioperative morbidity.
}

Amour BU Patel, Anna Reyes, Gareth L. Ackland

Background. Postoperative morbidity has immediate and delayed consequences for surgical patients, including excess risk of premature death. Capturing these data objectively and routinely in large electronic databases using tools such as the Postoperative Morbidity Survey (POMS) would offer tremendous clinical and translational potential. However, POMS has thus far only utilised prospective data collection by research staff. We hypothesised that retrospective data collection from routinely collated hospital data from paper and electronic charts, medical and nursing notes was non-inferior to prospective data collection requiring research staff capturing POMS-defined morbidity in real-time. Methods. Morbidity was recorded by a trained investigator as defined by POMS prospectively on postoperative days 3 and 7. Separately, an independent investigator blinded to prospectively acquired data retrospectively assessed the same patients' morbidity as defined by POMS criteria, using medical charts, nursing summaries and electronic data. Equivalence was accepted when the confidence limits for both modes of data collection fell completely inside the equivalence bounds, with the maximum equivalence difference (i.e. the largest value of the difference in sensitivities deemed to reach a conclusion of equivalence) set a priori at 0.2 . Differences for confidence limits between retrospective and prospective data collection were based on Nam's RMLE method. The relationship between morbidity on postoperative day 3 as recorded by each data collection method on time to become morbidity free and length of hospital stay was compared using the log-rank test. Results. POMS data from 85 patients undergoing elective or emergency surgery were analyzed. At postoperative day 3, POMS-defined morbidity was similar regardless of whether data were collected prospectively or retrospectively ( $95 \%$ confidence limits: -0.13 to $0.013 ; p<0.001$ ). Non-inferiority for sensitivity was observed for all other POMS domains and timepoints. Time to become morbidity free Kaplan-Meier plots were indistinguishable between POMS obtained prospectively or retrospectively (hazard ratio: 1.09 (95\% Cl: $0.76-1.57$ ); $p=0.33$, log rank test). Similarly, the mode of data collection did not alter the association between early postoperative morbidity on postoperative day 3 and delayed hospital discharge.

Conclusions. Postoperative morbidity as defined by the Post Operative Morbidity Survey 
can be assessed retrospectively. These data may therefore be easily captured using electronic patient record systems, thereby expanding the potential for bioinformatics approaches to generate new clinical and translational insights into recovery from surgery. 
1 Non-inferiority of retrospective data collection for assessing perioperative morbidity.

2 Amour B. U. Patel: Department of Anaesthesia, University College London Hospitals NHS

3 Trust, London, United Kingdom. UCL/UCLH National Institute for Health Research

4 Comprehensive Biomedical Research Centre, University College London Hospitals NHS Trust,

5 London, United Kingdom

6

7 Anna Reyes: UCL/UCLH National Institute for Health Research Comprehensive Biomedical

8 Research Centre, University College London Hospitals NHS Trust, London, United Kingdom.

9 Department of Anaesthesia, University College London Hospitals NHS Trust, London, United

10 Kingdom

11 Gareth L. Ackland: William Harvey Research Institute, Queen Mary University of London,

12 Charterhouse Square, London EC1M 6BQ United Kingdom. Department of Anaesthesia,

13 University College London Hospitals NHS Trust, London, United Kingdom. UCL/UCLH

14 National Institute for Health Research Comprehensive Biomedical Research Centre, University

15 College London Hospitals NHS Trust,

16 London, United Kingdom

18 Corresponding author Gareth L. Ackland: William Harvey Research Institute, Queen Mary

19 University of London, Charterhouse Square, London EC1M 6BQ United Kingdom.

20 Email: g.ackland@qmul.ac.uk 
23 Abstract

24 Background.

25 Postoperative morbidity has immediate and delayed consequences for surgical patients, including

26 excess risk of premature death. Capturing these data objectively and routinely in large electronic

27 databases using tools such as the Postoperative Morbidity Survey (POMS) would offer

28 tremendous clinical and translational potential. However, POMS has thus far only utilized

29 prospective data collection by research staff. We hypothesized that retrospective data collection

30 from routinely collated hospital data from paper and electronic charts, medical and nursing notes

31 was non-inferior to prospective data collection requiring research staff capturing POMS-defined

32 morbidity.

33 Methods.

34 Morbidity was recorded by a trained investigator as defined by POMS prospectively on

35 postoperative days 3 and 7. Separately, an independent investigator blinded to prospectively

36 acquired data retrospectively assessed the same patients' morbidity as defined by POMS criteria,

37 using medical charts, nursing summaries and electronic data. Equivalence was accepted when the

38 confidence limits for both modes of data collection fell completely inside the equivalence

39 bounds, with the maximum equivalence difference (i.e. the largest value of the difference in

40 sensitivities deemed to reach a conclusion of equivalence) set a priori at 0.2 . Differences for

41 confidence limits between retrospective and prospective data collection were based on Nam's

42 RMLE method. The relationship between morbidity on postoperative day 3 as recorded by each 
43 data collection method on time to become morbidity free and length of hospital stay was

44 compared using the log-rank test.

45 Results.

46 Results: POMS data from 85 patients undergoing elective or emergency surgery were analyzed.

47 At postoperative day 3, POMS-defined morbidity was similar regardless of whether data were

48 collected prospectively or retrospectively ( $95 \%$ confidence limits: -0.13 to $0.013 ; \mathrm{p}<0.001)$. Non-

49 inferiority for sensitivity was observed for all other POMS domains and time points. Time to

50 become morbidity free Kaplan-Meier plots were indistinguishable between POMS obtained

51 prospectively or retrospectively (hazard ratio: 1.09 (95\%CI: $0.76-1.57) ; \mathrm{p}=0.33$, log rank test).

52 Similarly, the mode of data collection did not alter the predictive value of early postoperative

53 morbidity on postoperative day 3 being associated with delayed hospital discharge.

54 Conclusions.

55 Postoperative morbidity as defined by the Post Operative Morbidity Survey can be assessed

56 retrospectively. These data may therefore be easily captured using electronic patient record

57 systems, thereby expanding the potential for bioinformatic approaches to generate new clinical

58 and translational insights into recovery from surgery. 
63 Introduction

64 The development of even minor postoperative complications is a major determinant of hospital readmission, long-term adverse outcomes and death. (Khuri et al. 2005; Moonesinghe et al. 2014). Postoperative morbidity can be recorded using a number of tools, but POMS (Post

67 Operative Morbidity Survey) has emerged as a useful survey for assessing short-term morbidity 68 following moderate-major surgery in clinical (Bennett-Guerrero et al. 1999; Grocott et al. 2007) 69 (Ackland et al. 2010; Ackland et al. 2015; Ackland et al. 2011; Ackland et al. 2007; Ausania et al. 2012; Davies et al. 2013; Jones et al. 2013; Pearse et al. 2014; Sanders et al. 2012; Snowden et al. 2010; Wakeling et al. 2005) and translational perioperative studies (Edwards et al. 2015). However, POMS has thus far only utilized prospective data collection, requiring research staff to record morbid events. The potential for electronic capture of these data is under-explored. However, determining whether retrospective, rather than prospective, data collection can capture POMS-defined morbidity is a first step that may help exploit these data for enhanced, large scale bioinformatic interrogation. We hypothesized that retrospective data collection from charts, medical and nursing notes was non-inferior to prospective data collection for capturing POMSdefined morbidity. We tested this by three different approaches. First, we established whether POMS- defined morbidity captured retrospectively was statistically non-inferior (Walker \& Nowacki 2011) to prospective, real time data collection, by calculating differences based on

81 Nam's RMLE method. (Nam 1997). Second, we assessed whether POMS-defined morbidity 82 captured retrospectively or prospectively altered the trajectory of patients becoming free of 83 postoperative morbidity. Third, we assessed whether POMS-defined morbidity on postoperative 84 day 3 captured retrospectively or prospectively was linked with delayed hospital discharge, the 
85 predictive value for which has been established in previous studies (Ackland et al. 2011).

87 Materials and Methods

88 We analysed POMS in 85 patients undergoing major elective surgery at University College

89 London Hospital, having obtained written informed consent (institutional board review- Medical

90 Research Ethics Committee: 10/WNo03/25). POMS domains are detailed in Table 1. Morbidity

91 was recorded as defined by POMS prospectively on postoperative days 3 and 7. Both

92 investigators were trained in prospectively collecting POMS data at the bedside. Thereafter, one

93 was assigned to prospective data collection at the bedside, while the other assessed POMS from

94 the same patients by retrospective analysis of medical charts, nursing summaries and electronic

95 data blinded to prospectively acquired data. The primary endpoint were whether retrospective

96 data collection demonstrated non-inferiority for sensitivity, compared to data recorded

97 prospectively for all-cause morbidity on postoperative day 3.

103 Table 1: POMS-defined morbidity. 
105 Morbidity type

106 Pulmonary: De novo requirement for supplemental oxygen or other respiratory support (e.g.,

107 continuous positive airway pressure or mechanical ventilation)

108 Infectious: Currently on antibiotics or temperature $>38^{\circ} \mathrm{C}$ in the last 24 hour

109 Renal: Presence of oliguria $(<500 \mathrm{~mL} /$ day $)$, increased serum creatinine $(>30 \%$ from baseline

110 value), or urinary catheter in place for a non-surgical reasons

111 Gastrointestinal: Unable to tolerate an enteral diet (either by mouth or feeding tube) for any

112 reason, including nausea, vomiting and abdominal distension

113 Cardiovascular: Diagnostic test or therapy in last 24 hours for any of the following reasons: de

114 novo myocardial infarction or ischemia, hypotension (requiring drug therapy or fluid $>200$

$115 \mathrm{~mL} / \mathrm{h}$ ), atrial or ventricular arrhythmia or pulmonary edema

116 Neurological: Presence of a de novo focal deficit, coma or confusion/delirium

117 Wound: Wound dehiscence requiring surgical exploration or drainage or pus from the wound

118 Hematological: Requirement for any of the following within last 24h: blood, platelets, fresh

119 frozen plasma or cryoprecipitate

120 Pain: Surgical wound pain significant enough to require parenteral opiates or regional anesthesia

121

122 Statistical Analysis 
123 Differences for confidence limits between retrospective and prospective data collection were

124 based on Nam's RMLE method. (Nam 1997). Maximum equivalence difference, the largest value

125 of the difference in sensitivities deemed to reach a conclusion of equivalence, was set a priori at

1260.2 (Walker \& Nowacki 2011). Equivalence was accepted when the confidence limits for both

127 modes of data collection fell completely inside the equivalence bounds. Alpha for testing the

128 hypothesis was set at 0.05 . Data are reported as mean $\pm \mathrm{SD}$, or confidence limits based on

129 Blackwelder and Nam's method. Time to become morbidity free was compared using the log-

130 rank test. The impact of morbidity on postoperative day 3 as defined by each data collection

131 method on length of hospital stay was compared using the log-rank test. P values $<0.05$ were

132 considered significant. NCSS 8 (NCSS, LLC. Kaysville, Utah, USA) was used for all statistical

133 analyses.

135 Power calculation

136 We calculated the sample size required to be 89 subjects, to achieve $80 \%$ power at a significance

137 level of 0.05 using a one-sided non-inferiority test of correlated proportions when the standard

138 proportion was 0.6 . The maximum ratio of these proportions that resulted in non-inferiority (the

139 range of non-inferiority) was set at 0.85 , and the actual ratio of the proportions was 1 (PASS 14

140 Power Analysis and Sample Size Software (2015). NCSS, LLC. Kaysville, Utah, USA).

141

142

143 Results 
144 The mean age of the cohort was $62 \pm 9 y ; 46 \%$ of patients were male. Eight colorectal, 22 vascular,

14529 orthopaedic and 26 urological surgical procedures were performed, lasting $2.4 \pm 1.4 \mathrm{~h}$. There

146 were no deaths during the hospital admission.

147

148 POMS-defined morbidity at any postoperative time point was identified in 52/85 (61\%) of

149 patients by prospective evaluation. POMS-defined morbidity was more common on

150 postoperative day 3 than 7 (Figure 1). Retrospective analysis similarly identified postoperative

151 morbidity on both postoperative days 3 and 7, with no domains beyond the upper bound

152 confidence limit for non-inferiority (Tables 2, 3).

153

154 Serial morbidity patterns.

155 Time to become morbidity free analysis (Figure 1) were indistinguishable based on data

156 collection by retrospective and prospective modes (hazard ratio: 1.09 (95\%CI: 0.76-1.57);

$157 \mathrm{p}=0.33$, by log rank test). 


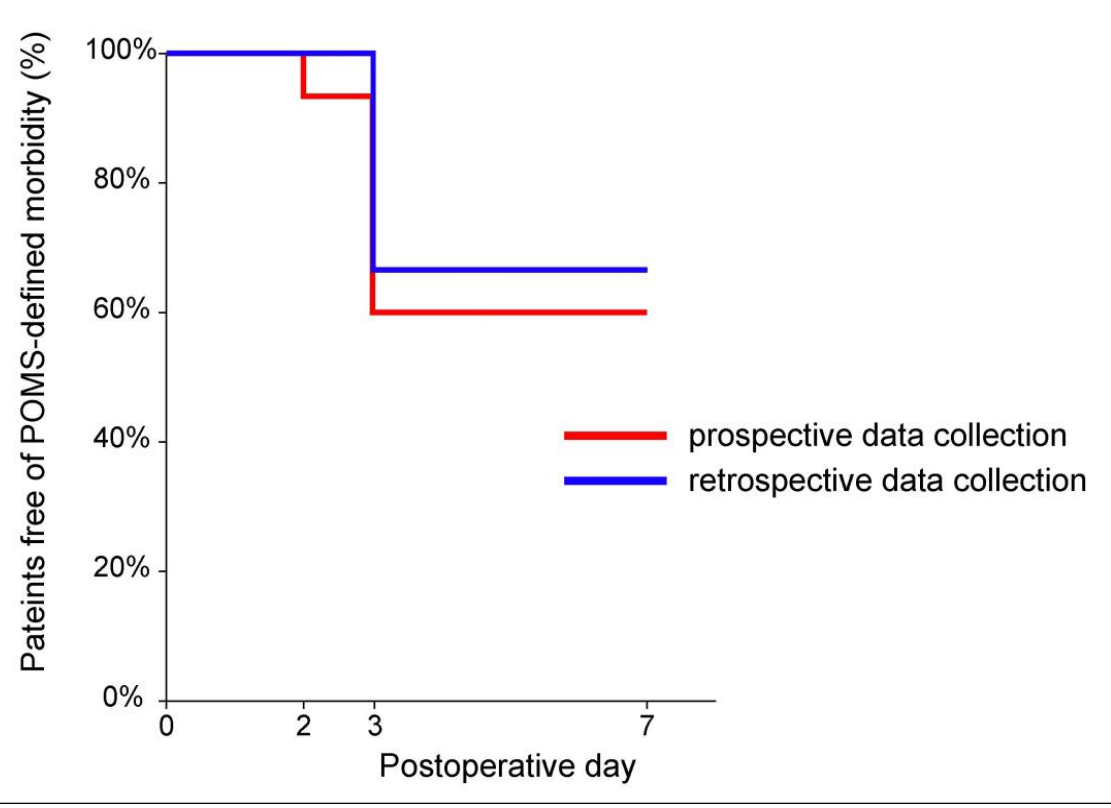

159 Figure 1. Kaplan-Meier plot of percentage of patients free of POMS-defined morbidity against

160 time by postoperative day. The plot demonstrates a similar percentage of patients were free of

161 postoperative morbidity at a given time-point through data collected retrospectively compared to

162 POMS data collected prospectively (hazard ratio: 1.09 (95\% CI: 0.76- 1.57); $\mathrm{p}=0.33$, by $\log$ rank 163 test).

164

165 Length of hospital stay

166 We have previously shown that the presence of POMS-defined morbidity on postoperative day 3

167 is associated with prolonged hospital stay. (Ackland et al. 2011) Both retrospective and

168 prospective modes of data collection that identified POMS-defined morbidity on postoperative

169 day 3 showed similar relationships with length of stay (Figure 2). 


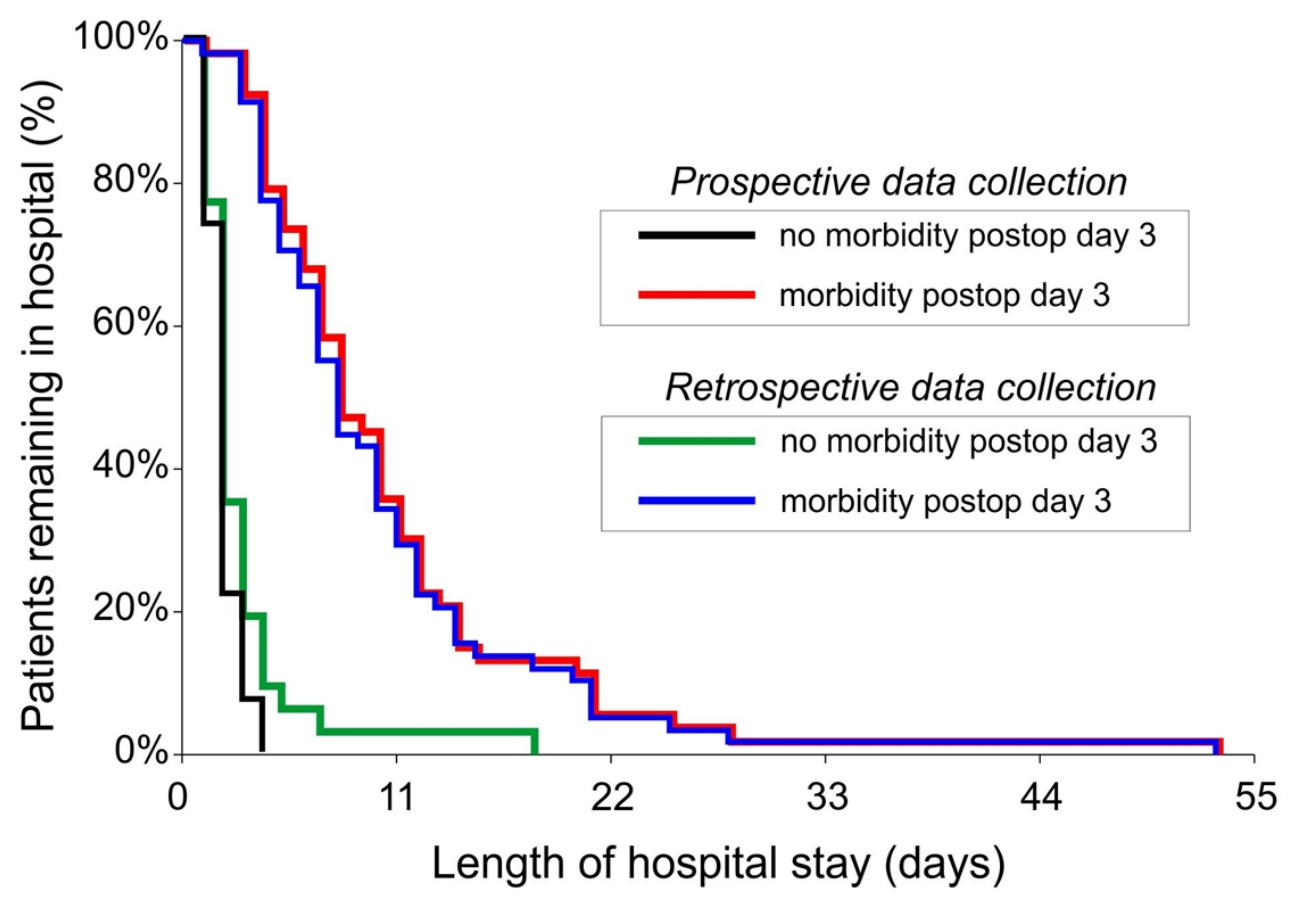

Figure 2.

171 Kaplan-Meier plot of percentage of patients remaining in hospital against time by length of

172 hospital stay. The plot demonstrates increased hospital length of stay in those sustaining POMS

173 on postoperative day 3, as recorded by both retrospective (hazard ratio: 5.0 (95\%CI: 2.3-10.6);

$174 \mathrm{p}<0.001$, by log rank test) and prospective (hazard ratio: 3.6 (95\%CI: $2.0-6.8) ; \mathrm{p}<0.001$, by $\log$

175 rank test) modes of data collection.

176

177 Table 2. Postoperative Day 3, equivalence and non-inferiority for sensitivity of mode of data

178 collection.

\begin{tabular}{|l|l|l|l|}
\hline Domain & P value & $\mathbf{9 5 \% C I}$ & Non-inferior? \\
\hline Any type & $<0.001$ & -0.13 to 0.013 & Yes \\
\hline
\end{tabular}




\begin{tabular}{|l|l|l|l|}
\hline Supplementary oxygen & $<0.001$ & -0.24 to 0.036 & Yes \\
\hline Antibiotics & $<0.001$ & -0.18 to 0.0039 & Yes \\
\hline Temperature & $<0.001$ & -0.058 to 0.0583 & Yes \\
\hline Urinary catheter & $<0.001$ & -0.086 to 0.0432 & Yes \\
\hline PONV & $<0.001$ & -0.14 to 0.0309 & Yes \\
\hline Enteral feeding & $<0.001$ & -0.058 to 0.0583 & Yes \\
\hline Confusion & $<$ & -0.058 to 0.0583 & Yes \\
\hline Products & $<0.001$ & -0.11 to to 0.058 & Yes \\
\hline Myocardial infarction & $<0.001$ & -0.058 to 0.0583 & Yes \\
\hline Arrhythmias & $<0.0001$ & -0.058 to 0.0583 & Yes \\
\hline & $<0018$ to 0.072 & \\
\hline & & -0.058 to 0.0583 & Yes \\
\hline & & & \\
\hline & & & \\
\hline
\end{tabular}




\begin{tabular}{|l|l|l|l|}
\hline & & & Yes \\
\hline Parenteral opioids & $<0.001$ & -0.2025 to 0.01 & Yes \\
\hline
\end{tabular}

180 Table 3. Postoperative Day 7, and non-inferiority for sensitivity of mode of data collection.

\begin{tabular}{|c|c|c|c|}
\hline Domain & $P$ value & $95 \% \mathrm{CI}$ & Non-inferior? \\
\hline Any type & 0.0002 & -0.17 to 0.04 & Yes \\
\hline Supplementary oxygen & 0.0008 & -0.088 to 0.088 & Yes \\
\hline Antibiotics & 0.0569 & 0.0036 to 0.2305 & Yes \\
\hline Temperature & 0.0004 & -0.13 to 0.065 & Yes \\
\hline Urinary catheter & 0.0089 & -0.042 to 0.17 & Yes \\
\hline PONV & 0.0008 & -0.088 to 0.088 & Yes \\
\hline Enteral feeding & 0.0002 & -0.17 to 0.042 & Yes \\
\hline Confusion & 0.0008 & -0.088 to 0.088 & Yes \\
\hline Myocardial infarction & 0.0004 & -0.1288 to 0.065 & Yes \\
\hline
\end{tabular}




\begin{tabular}{|l|l|l|l|}
\hline Arrhythmias & 0.0028 & -0.065 to 0.129 & Yes \\
\hline Drain & 0.0008 & -0.088 to 0.088 & Yes \\
\hline Packed red cells & 0.0004 & -0.13 to 0.065 & Yes \\
\hline Products & 0.0008 & -0.088 to 0.088 & Yes \\
\hline Pain & 0.0004 & -0.13 to 0.065 & Yes \\
\hline Parenteral opioids & 0.0008 & -0.088 to 0.088 & Yes \\
\hline
\end{tabular}

181

182 Discussion

183 This study shows that in patients undergoing elective or emergency surgery, retrospective

184 compilation of POMS-defined morbidity data from charts, medical and nursing notes is non-

185 inferior to prospective, real-time data collection requiring research or administrative personnel.

186 Our data demonstrate that similar proportions of patients were free of postoperative morbidity at

187 a given time-point through data captured retrospectively, compared to that collected

188 prospectively. POMS-defined morbidity on postoperative day 3 captured retrospectively and

189 prospectively was linked with similarly delayed hospital discharge. The non-inferiority of

190 retrospective data collection may provide significant economic benefit through reducing the need

191 for research staff collecting data in real-time. Furthermore, bioinformatic tools capable of mining 
192 clinical datasets should enable time-stamped, highly granular information on postoperative

193 morbidity. Our findings support the validity of future academic studies interrogating

194 postoperative data using this standardized retrospective approach.

195 Postoperative complications are critical predictors of long-term mortality, irrespective of

196 preoperative risk factors. (Khuri et al, 2004). Defining surgical patients at risk of delayed

197 adverse outcomes requires robust, sensitive measures of postoperative morbidity. Other systems

198 such as the Clavien-Dindo scale are tremendously useful for measuring deviations from usual

199 care and severity of postoperative morbidity. However, the Clavien-Dindo system, as with all

200 clinical assessment models, has had various shortcomings highlighted (Rassweiler et al. 2012).

201 Discriminating between surgical errors and apparently unforeseeable complications is

202 challenging. A urology study demonstrated that surgeons disagree widely on what

203 constitutes a complication for Clavien-Dindo grading. (de la Rosette et al. 2012)

205 There may be significant merit in combining systems to capture patient-centered outcomes

206 across the spectrum of low-high risk surgery and also to reflect severity of complications, as

207 recently utilized in a perioperative randomized controlled trial. (Ackland et al. 2015).

208 Irrespective of which system may be used, routine reporting of outcomes in noncardiac patient

209 population is limited; registry data has chiefly focused on technical and procedural outcomes.

210 POMS is an attractive tool as the survey questions are rapidly completed, have high inter-

211 observer agreement and are patient-centered outcomes. However, POMS to date has only utilized

212 prospectively collected data. The apparent need for prospective data collection not only makes

213 larger comparisons of reported outcomes challenging, but also hard to implement on a

214 widespread scale. This retrospective approach could therefore facilitate significant progress into 
215 expanding the number of patients for whom postoperative morbidity data can usefully be

216 collected, with a substantial impact on clinical and translational research work as a result.

217 Our data is consistent with other studies where POMS-defined morbidity is present in up to $75 \%$

218 of patients is associated with prolonged hospital stay. Although the data represents a single-

219 center case series, our post-hoc analysis of a well-validated, prospective descriptor of morbidity

220 (POMS) is the first study of its kind. A strength of this study is that bias was minimized through

221 data collection and retrospective analyses being performed by blinded independent investigators.

222 We demonstrate that postoperative data recorded prospectively can be attained and analyzed

223 using traditional systems of retrospective data collection. This certainly may reduce the costs of

224 postoperative morbidity data collection, and suggest their incorporation into electronic patient

225 records would be a surmountable software challenge. Length of hospital stay is increased by

226 'minor' postoperative complications (e.g. nausea and vomiting), which impacts on the financial

227 burden of medical healthcare. In this clinical setting, we have established a system to analyze

228 data in a more cost-effective way, to tackle this problem.

230 Conclusion

231 POMS-defined postoperative morbidity can be analyzed retrospectively. This approach suggests

232 that these data can be easily captured from electronic patient record systems, thereby expanding

233 the potential for bioinformatics approaches to generate new clinical and translational insights

234 into postoperative recovery. In this population, the non-inferiority of retrospective data collection

235 may contribute to real-time risk stratification, and warn of the true incidence and duration of

236 postoperative complications. 
239 References

240 Ackland GL, Harris S, Ziabari Y, Grocott M, and Mythen M. 2010. Revised cardiac risk index

241 and postoperative morbidity after elective orthopaedic surgery: a prospective cohort study. $\mathrm{Br} \mathrm{J}$

242 Anaesth 105:744-752. 10.1093/bja/aeq245 aeq245 [pii]

243 Ackland GL, Iqbal S, Paredes LG, Toner A, Lyness C, Jenkins N, Bodger P, Karmali S, Whittle

244 J, Reyes A, Singer M, Hamilton M, Cecconi M, Pearse RM, Mallett SV, Omar RZ, and group P-

245 Os. 2015. Individualised oxygen delivery targeted haemodynamic therapy in high-risk surgical

246 patients: a multicentre, randomised, double-blind, controlled, mechanistic trial. Lancet Respir

247 Med 3:33-41. 10.1016/S2213-2600(14)70205-X

248 Ackland GL, Moran N, Cone S, Grocott MP, and Mythen MG. 2011. Chronic kidney disease and

249 postoperative morbidity after elective orthopedic surgery. Anesth Analg 112:1375-1381.

250

10.1213/ANE.0b013e3181ee8456 ANE.0b013e3181ee8456 [pii]

251 Ackland GL, Scollay JM, Parks RW, de Beaux I, and Mythen MG. 2007. Pre-operative high

252 sensitivity C-reactive protein and postoperative outcome in patients undergoing elective

253 orthopaedic surgery. Anaesthesia 62:888-894. ANA5176 [pii] 10.1111/j.1365-

$254 \quad 2044.2007 .05176 . x$

255 Ausania F, Snowden CP, Prentis JM, Holmes LR, Jaques BC, White SA, French JJ, Manas DM, 256 and Charnley RM. 2012. Effects of low cardiopulmonary reserve on pancreatic leak following

257 pancreaticoduodenectomy. Br J Surg 99:1290-1294. 10.1002/bjs.8859

258 Bennett-Guerrero E, Welsby I, Dunn TJ, Young LR, Wahl TA, Diers TL, Phillips-Bute BG, 
259 Newman MF, and Mythen MG. 1999. The use of a postoperative morbidity survey to evaluate

260 patients with prolonged hospitalization after routine, moderate-risk, elective surgery.

261 AnesthAnalg 89:514-519.

262 Davies SJ, Francis J, Dilley J, Wilson RJ, Howell SJ, and Allgar V. 2013. Measuring outcomes 263 after major abdominal surgery during hospitalization: reliability and validity of the Postoperative 264 Morbidity Survey. Perioper Med (Lond) 2:1. 10.1186/2047-0525-2-1

265 de la Rosette JJ, Opondo D, Daels FP, Giusti G, Serrano A, Kandasami SV, Wolf JS Jr, Grabe

266 M, Gravas S, Croes Pcnl Study Group. 2012. Categorisation of complications and validation of

267 the Clavien score for percutaneous nephrolithotomy. Eur Urol. 62: 246-55.

268

269 Edwards MR, Sultan P, Gutierrez Del Arroyo A, Whittle J, Karmali SN, Moonesinghe SR,

270 Haddad FS, Mythen MG, Singer M, and Ackland GL. 2015. Metabolic dysfunction in

271 lymphocytes promotes postoperative morbidity. Clinical science in press. CS20150024 [pii]

$272 \quad 10.1042 / \mathrm{CS} 20150024$

273 Grocott MP, Browne JP, Van der MJ, Matejowsky C, Mutch M, Hamilton MA, Levett DZ,

274 Emberton M, Haddad FS, and Mythen MG. 2007. The Postoperative Morbidity Survey was

275 validated and used to describe morbidity after major surgery. JClinEpidemiol 60:919-928.

276 Jones C, Kelliher L, Dickinson M, Riga A, Worthington T, Scott MJ, Vandrevala T, Fry CH,

277 Karanjia N, and Quiney N. 2013. Randomized clinical trial on enhanced recovery versus

278 standard care following open liver resection. Br J Surg 100:1015-1024. 10.1002/bjs.9165

279 Khuri SF, Henderson WG, DePalma RG, Mosca C, Healey NA, and Kumbhani DJ. 2005. 
280 Determinants of long-term survival after major surgery and the adverse effect of postoperative 281 complications. AnnSurg 242:326-341.

282 Moonesinghe SR, Harris S, Mythen MG, Rowan KM, Haddad FS, Emberton M, and Grocott 283 MP. 2014. Survival after postoperative morbidity: a longitudinal observational cohort 284 studydagger. Br J Anaesth. aeu224 [pii] 10.1093/bja/aeu224

285 Nam JM. 1997. Establishing equivalence of two treatments and sample size requirements in 286 matched-pairs design. Biometrics 53:1422-1430.

287 Pearse RM, Harrison DA, MacDonald N, Gillies MA, Blunt M, Ackland GL, Grocott MP, Ahern 288 A, Griggs K, Scott R, Hinds C, and Rowan K. 2014. Effect of a perioperative, cardiac output289 guided hemodynamic therapy algorithm on outcomes following major gastrointestinal surgery: a 290 randomized clinical trial and systematic review. JAMA 311:2181-2190. 10.1001/jama.2014.5305

$291 \quad 1873985$ [pii]

292 Rassweiler JJ, Rassweiler MC, and Michel MS. 2012. Classification of complications: is the 293 Clavien- Dindo classification the gold standard? European urology 62:256-258; discussion 259294 260. 10.1016/j.eururo.2012.04.028 S0302-2838(12)00494-0 [pii]

295 Sanders J, Keogh BE, Van der Meulen J, Browne JP, Treasure T, Mythen MG, and Montgomery 296 HE. 2012. The development of a postoperative morbidity score to assess total morbidity burden 297 after cardiac surgery. J Clin Epidemiol 65:423-433. 10.1016/j.jclinepi.2011.11.004 S0895298 4356(11)00363-5 [pii]

299 Snowden CP, Prentis JM, Anderson HL, Roberts DR, Randles D, Renton M, and Manas DM. 
300 2010. Submaximal cardiopulmonary exercise testing predicts complications and hospital length

301 of stay in patients undergoing major elective surgery. AnnSurg 251:535-541.

302 Wakeling HG, McFall MR, Jenkins CS, Woods WG, Miles WF, Barclay GR, and Fleming SC.

303 2005. Intraoperative oesophageal Doppler guided fluid management shortens postoperative

304 hospital stay after major bowel surgery. Br J Anaesth 95:634-642. 10.1093/bja/aei223

305 Walker E, and Nowacki AS. 2011. Understanding equivalence and noninferiority testing. J Gen

306 Intern Med 26:192-196. 10.1007/s11606-010-1513-8. 